\title{
An Improved, Fully Heterogeneous, Diastereoselective Synthesis of (Z)-a-Bromonitroalkenes
}

\author{
Alessandro Palmieri, Serena Gabrielli, Roberto Ballini* \\ Green Chemistry Group, School of Science and Technology, Chemistry Division, University of Camerino, Via S. Agostino 1, 62032 Camerino \\ (MC), Italy \\ Fax +39(0737)402297; E-mail: roberto.ballini@unicam.it \\ Received: 30.10.2012; Accepted: 02.11.2012
}

\begin{abstract}
Bromonitroalkenes are both key starting materials for the preparation of complex structures and possess antimicrobial activity. In this context, we disclose a simple, fully heterogeneous synthetic approach for their preparation in good overall yields.
\end{abstract}

Key words: Henry reaction, dehydration, diastereoselectivity, $\alpha$-bromonitroalkanes, heterogeneous catalysis

Unsaturated nitro compounds are an important class of valuable precursors to a wide variety of target molecules. ${ }^{1}$ The utility of conjugated nitroalkenes in organic synthesis is largely due to their versatile reactivity as Michael acceptors, dipolarophiles, 1,3-dipoles, dienophiles, and heterodienes. ${ }^{2}$ Nitroalkenes are also distinguished by their diverse biological activities. ${ }^{3}$ In this respect, of particular interest are the $\alpha$-bromonitroalkenes $\mathbf{5}$ since they have shown important antimicrobial activities ${ }^{4}$ as well as being key intermediates. ${ }^{5}$

Due to the importance of these compounds, their easy availability is strategically important and simpler synthetic procedures for their preparation would increase the importance of these bifunctionalized alkenes. The general synthetic method for the preparation of $\alpha$-bromonitroalkenes involves bromination-debromination of nitroalkenes 3 (Scheme 1). However, since nitroalkanes 3 are generated by Henry reaction followed by elimination, it is clear that the preparation of $\alpha$-bromonitroalkenes 5 starting from their ultimate precursors (nitromethane and aldehydes) involves a complex multistep sequence. ${ }^{6}$

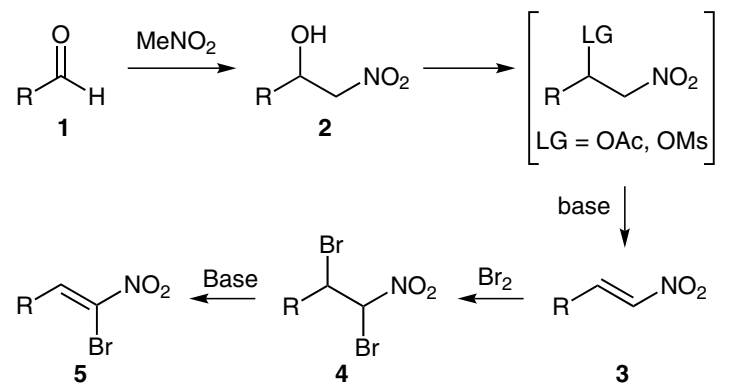

Scheme 1

SYNLETT 2013, 24, 0114-0116

Advanced online publication: 28.11 .2012

DOI: 10.1055/s-0032-1317695; Art ID: ST-2012-D0929-L

(C) Georg Thieme Verlag Stuttgart · New York
Some decades ago, a direct procedure ${ }^{7}$ involving the nitroaldol condensation of aldehydes with bromonitromethane in the presence of tri- $n$-butylarsine was described. However, the method appears to work just with aromatic aldehydes, and requires a large excess of bromonitromethane, a dry atmosphere, and high temperatures. Moreover, the reaction must be manipulated with attention in order to avoid possible explosion, probably due to the use of tri- $n$ butylarsine. Thus, a simpler method would be valuable. In this context, based on our previous experience in the nitroaldol (Henry) reaction, ${ }^{8}$ we have developed a new, simple, mild, and fully heterogeneous approach for the synthesis of $\alpha$-bromonitroalkenes 5 starting from the Henry reaction between aldehydes $\mathbf{1}$ and bromonitromethane $\mathbf{6}$, followed by the dehydration of the obtained crude nitro alcohols (Scheme 2).

We chose, as a model system, the reaction of 6 with hexanal (1a) under basic and solvent-free conditions (carbonate on silica), in which the crude nitroalkanol 7a can be directly dehydrated, by Amberlyst $15 / \mathrm{Ac}_{2} \mathrm{O}^{9}$ into the target compound $\mathbf{5 a}$ avoiding any intermediate purification step.

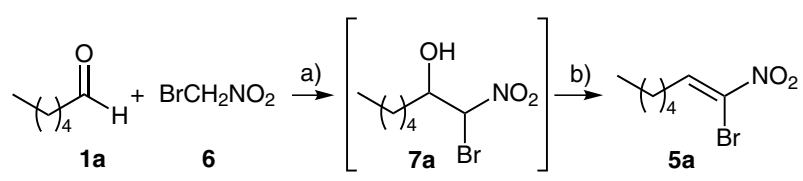

Scheme 2 Reagents and conditions: a) SolFC, carbonate on silica, r.t., 4.5 h; b) Amberlyst 15, EtOAc, r.t., 1.5 h.

As reported in Table 1, the optimal result was obtained by employing 0.3 equivalents of carbonate on silica, coupled with $500 \mathrm{mg} / \mathrm{mmol}$ of Amberlyst 15 and three equivalents of $\mathrm{Ac}_{2} \mathrm{O}$.

In order to assess the generality of our procedure we investigated a variety of substrates and, as reported in Table 2 , satisfactory to good yields (55-85\%) were achieved with aromatic and aliphatic aldehydes, including functionalized substrates. ${ }^{10}$ Only 5 -bromo-2-furfural $\mathbf{1 k}$ (Table 2 , entry 11$)$ afforded a low yield (31\%) of alkene $\mathbf{5 k}$, probably due to the chemical frailty of the furan ring.

Furthermore, all the obtained $\alpha$-bromonitroalkenes were isolated as a single $Z$-diastereomer. The configuration was established by comparison with the literature data. ${ }^{4 \mathrm{~d}, 6 \mathrm{c}}$ 
Table 1

\begin{tabular}{lllll}
\hline Entry & $\begin{array}{l}\text { Carbonate on } \\
\text { silica (equiv) }\end{array}$ & $\begin{array}{l}\text { Amberlyst } 15 \\
(\mathrm{~g} / \mathrm{mmol})\end{array}$ & $\begin{array}{l}\mathrm{Ac}_{2} \mathrm{O} \\
\text { (equiv) }\end{array}$ & $\begin{array}{l}\text { Yield of 5a } \\
(\%)^{\mathrm{a}}\end{array}$ \\
\hline 1 & 0.1 & 0.25 & 2 & 20 \\
2 & 0.1 & 0.35 & 2 & 31 \\
3 & 0.1 & 0.5 & 2 & 48 \\
4 & 0.1 & 1.0 & 2 & 50 \\
5 & 0.1 & 0.5 & 3 & 56 \\
6 & 0.1 & 0.5 & 4 & 54 \\
7 & 0.2 & 0.5 & 3 & 67 \\
8 & 0.3 & 0.5 & 3 & 82 \\
9 & 0.4 & 0.5 & 3 & 81 \\
10 & 0.3 & - & 3 & no product \\
11 & 0.3 & 0.5 & - & no product \\
\hline
\end{tabular}

${ }^{\text {a }}$ Yield of pure isolated product.

Table 2

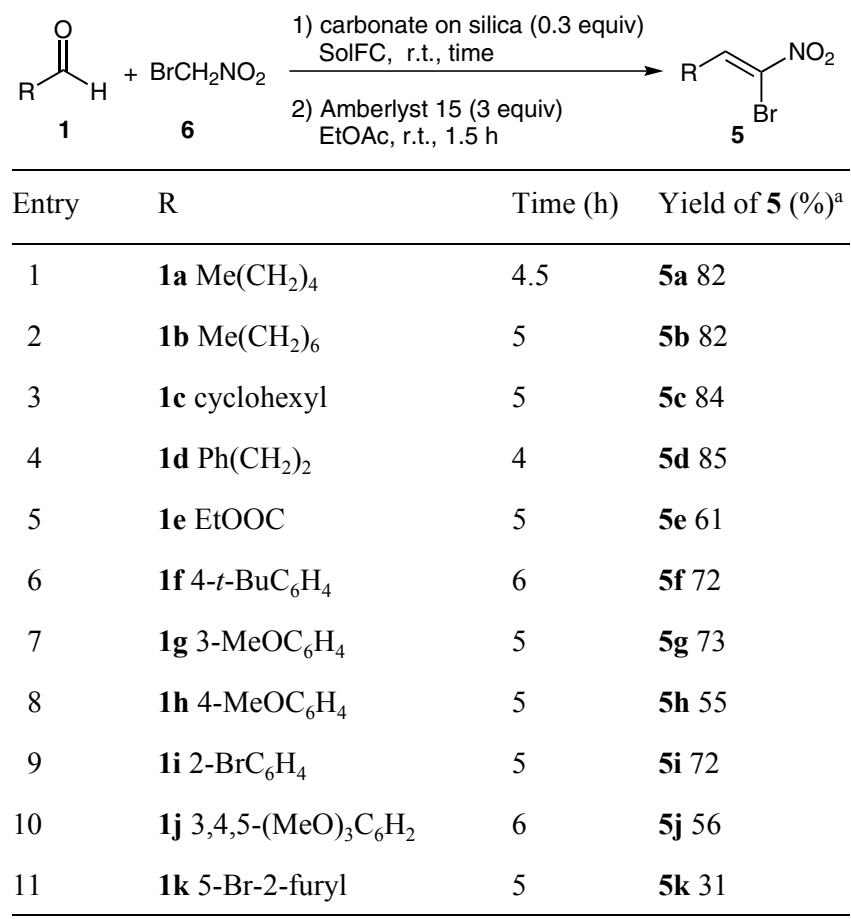

${ }^{\text {a }}$ Yield of pure isolated product.

In conclusion, our procedure offers important advantages with respect to the previous reported approaches since it gives access to the target compounds, without the need for excess bromonitromethane, under mild reaction conditions (room temperature) and short reaction times, with evident economical and environmental benefits. Moreover, a variety of other important functionalities can be tolerated giving access to polyfunctionalized $\alpha$-bromoni- troalkenes that could be of interest as targets with potential biological activities. ${ }^{4}$

Finally, it is important to point out that our method employs a fully heterogeneous procedure and that the final dehydration of nitroalkanol proceeds under acidic conditions, contrary to the standard procedures that usually employ basic conditions.

\section{Acknowledgment}

The authors thank the University of Camerino and MIUR, Italy (FIRB National Project 'Metodologie di nuova generazione nella formazione di legami carbonio-carbonio e carbonio-eteroatomo in condizioni eco-sostenibili') for financial support.

\section{References and Notes}

(1) (a) Perekalin, V. V.; Lipina, E. S.; Berestovitskaya, V. M.; Efremov, D. A. In Nitroalkenes Conjugated Nitro Compounds; Wiley: Chichester, 1994. (b) Ballini, R. In Studies in Natural Products Chemistry; Atta-ur-Rahman, Elsevier: Amsterdam, 1997, 117. (c) Ballini, R.; Marcantoni, E.; Petrini, M. In Amino Group Chemistry; Ricci, A., Ed.; Wiley-VCH: Weinheim, 2008, 93. (d) Ballini, R.; Gabrielli, S.; Palmieri, A. Curr. Org. Chem. 2010, 14,65 .

(2) See, for example: (a) Berner, O. M.; Tedeschi, L.; Enders, D. Eur. J. Org. Chem. 2002, 1877. (b) Krause, N.; HoffmannRöder, A. Synthesis 2001, 171. (c) Denmark, S. E.; Thorarensen, A. Chem. Rev. 1996, 96, 137. (d) Tietze, L. F.; Kettschau, G. Top. Curr. Chem. 1997, 189, 1. (e) Namboothiri, I. N. N.; Hassner, A. Top. Curr. Chem. 2001, 216, 1 .

(3) (a) Kabalka, G. W.; Varma, R. S. Org. Prep. Proced. Int. 1987, 19, 283. (b) Barret, A. G. M. Chem. Soc. Rev. 1991, 20, 95. (c) Blades, K.; Butt, A. H.; Cockerill, G. S.; Easterfield, H. J.; Lequeux, T. P.; Percy, J. M. J. Chem. Soc., Perkin Trans. 1 1999, 3609. (d) Hoashi, Y.; Yabuta, T.; Takemoto, Y. Tetrahedron Lett. 2004, 45, 9185. (e) Dadwal, M.; Mohan, R.; Panda, D.; Mobin, S. M.; Namboothiri, I. N. N. Chem. Commun. 2006, 338. (f) Rastogi, N.; Mohan, R.; Panda, D.; Mobin, S. M.; Namboothiru, I. N. N. Org. Biomol. Chem. 2006, 4, 3211. (g) Mohan, R.; Rastogi, N.; Namboothiri, I. N. N.; Mobin, S. M.; Panda, D. Bioorg. Med. Chem. 2006, 14, 8073. (h) Parry, R.; Nishino, S.; Spain, J. Nat. Prod. Rep. 2011, 28, 152.

(4) See, for example: (a) Cancio, C.; Ramon, N.; Placeres, G.; Exiquio, T. WO 0153283, 2001. (b) McCoy, W. F.; Thornburgh, S. WO 8911793, 1989. (c) González-Diaz, H.; Tenorio, E.; Castañedo, N.; Santana, L.; Uriarte, E. Bioorg. Med. Chem. 2005, 13, 1523. (d) Estrada, E.; Gómez, M.; Castañedo, N.; Pérez, C. J. Mol. Struct. (Theochem) 1999, $468,193$.

(5) (a) Ganesh, M.; Namboothiri, I. N. N. Tetrahedron 2007, 63, 11973. (b) McCooey, S. H.; McCabe, T.; Connon, S. J. J. Org. Chem. 2006, 71, 7494. (c) Romashov, L. V.; Khomutova, Y. A.; Danilenko, V. M.; Ioffe, S. L.; Lesiv, A. V. Synthesis 2010, 407.

(6) See, for example: (a) Sarkisyan, Z. M.; Sadikov, K. D.; Smirnov, A. S.; Kuzhaeva, A. A.; Makarenko, S. V.; Anisimova, N. A.; Deiko, L. I.; Berestovitskaya, V. M.; Russian, V. M. J. Org. Chem. 2004, 40, 908. (b) Tuan, D. T.; Tung, D. T.; Langer, P. Synlett 2006, 2812. (c) Ganesh, M.; Namboothiri, I. N. N. Tetrahedron 2007, 63, 11973.

(7) Shen, Y.; Yang, B. Synth. Commun. 1993, 23, 1. 
(8) (a) Ballini, R.; Castagnani, R.; Petrini, M. J. Org. Chem. 1992, 57, 2160. (b) Ballini, R.; Bosica, G.; Forconi, P. Tetrahedron 1996, 52, 1677. (c) Ballini, R.; Bosica, G. J. Org. Chem. 1997, 62, 425. (d) Ballini, R.; Bosica, G.; Parrini, M. Chem. Lett. 1999, 1105. (e) Ballini, R.; Bigi, F.; Giorgi, E.; Maggi, R.; Sartori, G. J. Catal. 2000, 191, 348. (f) Ballini, R.; Bosica, G.; Livi, D.; Palmieri, A.; Maggi, R.; Sartori, G. Tetrahedron Lett. 2003, 44, 2271. (g) Ballini, R.; Fiorini, D.; Gil, M. V.; Palmieri, A. Tetrahedron 2004, 60, 2799. (h) Ballini, R.; Barboni, L.; Palmieri, A. Synlett 2007, 3019. (i) Ballini, R.; Bosica, G.; Palmieri, A.; Pizzo, F.; Vaccaro, L. Green Chem. 2008, 10, 541. (j) Ballini, R.; Noé, M.; Perosa, A.; Selva, M. J. Org. Chem. 2008, 73, 8520.

(9) Usually, the dehydration of bromonitroalkanols is performed under basic conditions, see ref. 6 .

(10) Typical Procedure for the Synthesis of Compounds 5 The catalyst $(0.56 \mathrm{~g}, 0.3 \mathrm{mmol})$ was slowly added at r.t. to a mechanically stirred mixture of the bromonitromethane $(6,1$ mmol, $0.155 \mathrm{~g}$, pure $90 \%$ ) and the requisite aldehyde $\mathbf{1}(1$ $\mathrm{mmol}$ ), and the reaction was stirred for the appropriate time (see Table). The mixture was then treated with EtOAc (5 $\mathrm{mL}$ ), and the catalyst was filtered off and washed with additional EtOAc $(10 \mathrm{~mL})$. The solution was concentrated under reduced pressure to a volume of $3 \mathrm{~mL}$ and then treated with $\mathrm{Ac}_{2} \mathrm{O}(306 \mathrm{mg}, 3 \mathrm{mmol})$ and Amberlyst $15(0.5 \mathrm{~g})$ and stirred, at r.t., for an additional $1.5 \mathrm{~h}$. Finally, the Amberlyst 15 was removed by filtration, washing with EtOAc $(7 \mathrm{~mL})$, and the solution was concentrated under vacuum giving the crude product 5 which was purified by flash chromatography (hexane-EtOAc $=9: 1$ ).

\section{(Z)-1-Bromo-1-nitrohept-1-ene (5a)}

Light yellow oil. IR (neat): $v=3045,1551,1325,1261,942$, $722 \mathrm{~cm}^{-1}$. ${ }^{1} \mathrm{H}$ NMR $\left(400 \mathrm{MHz}, \mathrm{CDCl}_{3}\right): \delta=0.91(\mathrm{t}, 3 \mathrm{H}, J=$ $6.8 \mathrm{~Hz}), 1.30-1.40$ (m, $4 \mathrm{H}), 1.51-1.61(\mathrm{~m}, 2 \mathrm{H}), 2.37$ (q, 2 $\mathrm{H}, J=7.3 \mathrm{~Hz}), 7.67(\mathrm{t}, 1 \mathrm{H}, J=7.3 \mathrm{~Hz}) .{ }^{13} \mathrm{C} \mathrm{NMR}(100 \mathrm{MHz}$, $\left.\mathrm{CDCl}_{3}\right): \delta=14.1,22.5,27.3,31.3,31.6,131.2,141.6 . \mathrm{MS}$ $(\mathrm{EI}, 70 \mathrm{eV}): m / z(\%)=165,142,119,95,69,55,41(100)$, 39, 30. Anal. Calcd for $\mathrm{C}_{7} \mathrm{H}_{12} \mathrm{BrNO}_{2}$ (222.08): C, 37.86; $\mathrm{H}$, $5.45 ; \mathrm{N}, 6.31$. Found: C, 37.91; H, 5.49; N, 6.28.

(Z)-1-Bromo-1-nitronon-1-ene (5b)

Light yellow oil. IR (neat): $v=3040,1542,1321,1254,960$, $768 \mathrm{~cm}^{-1}$. ${ }^{1} \mathrm{H} \mathrm{NMR}\left(400 \mathrm{MHz}, \mathrm{CDCl}_{3}\right): \delta=0.89(\mathrm{t}, 3 \mathrm{H}, J=$ $6.8 \mathrm{~Hz}), 1.23-1.41(\mathrm{~m}, 8 \mathrm{H}), 1.50-1.61(\mathrm{~m}, 2 \mathrm{H}), 2.37$ (q, 2 $\mathrm{H}, J=7.3 \mathrm{~Hz}), 7.66(\mathrm{t}, 1 \mathrm{H}, J=7.3 \mathrm{~Hz}) \cdot{ }^{13} \mathrm{C}$ NMR $(100 \mathrm{MHz}$, $\left.\mathrm{CDCl}_{3}\right): \delta=14.3,22.8,27.6,29.1,29.4,31.3,31.9,131.2$, 141.6. MS (EI, $70 \mathrm{eV}): m / z(\%)=234,121,93,81,67,55$, 43 (100), 41, 39, 29. Anal. Calcd for $\mathrm{C}_{9} \mathrm{H}_{16} \mathrm{BrNO}_{2}$ (250.13): C, 43.22; H, 6.45; N, 5.60. Found: C, 43.27; H, 6.49; N, 5.58 . (Z)-(2-Bromo-2-nitrovinyl)cyclohexane (5c)

Light yellow oil. IR (neat): $v=3030,1539,1320,971,960$, $761 \mathrm{~cm}^{-1}$. ${ }^{1} \mathrm{H}$ NMR $\left(400 \mathrm{MHz}, \mathrm{CDCl}_{3}\right): \delta=1.18-1.44(\mathrm{~m}, 6$ $\mathrm{H}), 1.65-1.84(\mathrm{~m}, 4 \mathrm{H}), 2.39-2.53(\mathrm{~m}, 1 \mathrm{H}), 7.48$ (d, $1 \mathrm{H}, J$ $=9.8 \mathrm{~Hz}) \cdot{ }^{13} \mathrm{CNMR}\left(100 \mathrm{MHz}, \mathrm{CDCl}_{3}\right): \delta=25.3,25.7,30.8$, 40.8, 129.7, 145.3. MS (EI, $70 \mathrm{eV}): m / z(\%)=218,216,137$, $119,97,91,81,79,71,69,55(100), 41,39$. Anal. Calcd for $\mathrm{C}_{8} \mathrm{H}_{12} \mathrm{BrNO}_{2}$ (234.09): C, 41.05; H, 5.17; N, 5.98. Found: $\mathrm{C}$, 41.09; H, 5.21; N, 5.96.

\section{(Z)-(4-Bromo-4-nitrobut-3-enyl)benzene (5d)}

Light yellow oil. IR (neat): $v=3035,1618,1525,1331,937$, $750,724,698 \mathrm{~cm}^{-1}$. ${ }^{1} \mathrm{H}$ NMR (400 MHz, $\left.\mathrm{CDCl}_{3}\right): \delta=2.71$ (q, $2 \mathrm{H}, J=7.7 \mathrm{~Hz}), 2.88(\mathrm{t}, 2 \mathrm{H}, J=7.7 \mathrm{~Hz}), 7.17-7.36(\mathrm{~m}$, $5 \mathrm{H}), 7.67(\mathrm{t}, 1 \mathrm{H}, J=7.7 \mathrm{~Hz}) .{ }^{13} \mathrm{C} \mathrm{NMR}\left(100 \mathrm{MHz}, \mathrm{CDCl}_{3}\right)$ : $\delta=33.0,33.6,127.0,128.5,129.0,131.8,139.7,140.2$. MS $(E I, 70 \mathrm{eV}): m / z(\%)=240,238,129,91(100), 65$. Anal. Calcd for $\mathrm{C}_{10} \mathrm{H}_{10} \mathrm{BrNO}_{2}(256.10): \mathrm{C}, 46.90 ; \mathrm{H}, 3.94 ; \mathrm{N}, 5.47$. Found: C, 46.88; H, 3.97; N, 5.44 .
(Z)-Ethyl 3-Bromo-3-nitroacrylate (5e)

Light yellow oil. IR (neat): $v=1733,1622,1552,1323 \mathrm{~cm}$ ${ }^{1} .{ }^{1} \mathrm{H}$ NMR $\left(400 \mathrm{MHz}, \mathrm{CDCl}_{3}\right): \delta=1.35(\mathrm{t}, 3 \mathrm{H}, J=7.3 \mathrm{~Hz})$, $4.34(\mathrm{q}, 2 \mathrm{H}, J=7.3 \mathrm{~Hz}), 7.74(\mathrm{~s}, 1 \mathrm{H}) .{ }^{13} \mathrm{C}$ NMR $(100 \mathrm{MHz}$, $\left.\mathrm{CDCl}_{3}\right): \delta=14.2,62.8,125.8,139.3,162.2$. MS (EI, $\left.70 \mathrm{eV}\right)$ : $m / z(\%)=180,178,166,164,151,149,133,131,106,104$, 69, 53, 29 (100). Anal. Calcd for $\mathrm{C}_{5} \mathrm{H}_{6} \mathrm{BrNO}_{4}(224.01)$ : $\mathrm{C}$, 26.81; H, 2.70; N, 6.25. Found: C, 26.86; H, 2.72; N, 6.22. (Z)-1-(2-Bromo-2-nitrovinyl)-4-tert-butylbenzene (5f) Yellow solid, $\mathrm{mp} 50-52{ }^{\circ} \mathrm{C}$. IR (neat): $v=3030,1599,1530$, $1325,953,834,762 \mathrm{~cm}^{-1} .{ }^{1} \mathrm{H}$ NMR $\left(400 \mathrm{MHz}, \mathrm{CDCl}_{3}\right): \delta=$ $1.35(\mathrm{~s}, 9 \mathrm{H}), 7.51(\mathrm{~d}, 2 \mathrm{H}, J=8.5 \mathrm{~Hz}), 7.86(\mathrm{~d}, 2 \mathrm{H}, J=8.5$ $\mathrm{Hz}), 8.64(\mathrm{~s}, 1 \mathrm{H}) .{ }^{13} \mathrm{C} \mathrm{NMR}\left(100 \mathrm{MHz}, \mathrm{CDCl}_{3}\right): \delta=31.3$, $35.4,126.3,127.3,127.5,131.3,136.7,156.3$. MS (EI, 70 $\mathrm{eV}): m / z(\%)=285,283,270,268,222,220,143(100), 128$, 115, 57. Anal. Calcd for $\mathrm{C}_{12} \mathrm{H}_{14} \mathrm{BrNO}_{2}(284.15)$ : C, 50.72; H, 4.97; N, 4.93. Found: C, 50.76; H, 5.00; N, 4.90.

(Z)-1-(2-Bromo-2-nitrovinyl)-3-methoxybenzene (5g) Yellow solid, $\mathrm{mp} 68-70{ }^{\circ} \mathrm{C}$. IR (neat): $v=3070,3029,1607$, 1553, 1331, 1176, 897, $743 \mathrm{~cm}^{-1} .{ }^{1} \mathrm{H}$ NMR $(400 \mathrm{MHz}$, $\left.\mathrm{CDCl}_{3}\right): \delta=3.86(\mathrm{~s}, 3 \mathrm{H}), 7.07(\mathrm{~d}, 1 \mathrm{H}, J=7.7 \mathrm{~Hz}), 7.38$

$7.46(\mathrm{~m}, 3 \mathrm{H}), 8.61(\mathrm{~s}, 1 \mathrm{H}) .{ }^{13} \mathrm{C} \mathrm{NMR}\left(100 \mathrm{MHz}, \mathrm{CDCl}_{3}\right): \delta$ $=55.6,115.8,118.0,124.0,128.5,130.2,131.5,136.6$, 159.9. MS (EI, $70 \mathrm{eV}): m / z(\%)=259,257,212,210,150$, $148,132(100), 102,89,77,63$. Anal. Calcd for $\mathrm{C}_{9} \mathrm{H}_{8} \mathrm{BrNO}_{3}$ (258.07): C, 41.89; H, 3.12; N, 5.43. Found: C, 41.93; H, $3.10 ; \mathrm{N}, 5.40$.

(Z)-1-(2-Bromo-2-nitrovinyl)-4-methoxybenzene (5h)

Yellow solid, $m p 59-61{ }^{\circ} \mathrm{C}$. IR (neat): $v=3041,1520,1310$, 1261, 1181, 957, $825 \mathrm{~cm}^{-1} .{ }^{1} \mathrm{H}$ NMR (400 MHz, $\left.\mathrm{CDCl}_{3}\right): \delta$ $=3.88(\mathrm{~s}, 3 \mathrm{H}), 7.00(\mathrm{~d}, 2 \mathrm{H}, J=8.5 \mathrm{~Hz}), 7.92(\mathrm{~d}, 2 \mathrm{H}, J=8.5$ $\mathrm{Hz}), 8.63$ (s, $1 \mathrm{H}) .{ }^{13} \mathrm{C} \mathrm{NMR}\left(100 \mathrm{MHz}, \mathrm{CDCl}_{3}\right): \delta=55.8$, 114.8, 122.6, 125.6, 133.7 136.6, 163.0. MS (EI, $70 \mathrm{eV}$ ): $m / z(\%)=259,257,212,210,132(100), 117,89,63$. Anal. Calcd for $\mathrm{C}_{9} \mathrm{H}_{8} \mathrm{BrNO}_{3}(258.07)$ : C, 41.89; H, 3.12; N, 5.43 . Found: C, 41.93; H, 3.09; N, 5.45.

(Z)-1-Bromo-2-(2-bromo-2-nitrovinyl)benzene (5i)

Yellow waxy solid. IR (neat): $v=3078,3035,1606,1582$, $1532,1300,898,764,733,703 \mathrm{~cm}^{-1} .{ }^{1} \mathrm{H}$ NMR $(400 \mathrm{MHz}$, $\left.\mathrm{CDCl}_{3}\right): \delta=7.35(\mathrm{t}, 1 \mathrm{H}, J=7.7 \mathrm{~Hz}), 7.44(\mathrm{t}, 1 \mathrm{H}, J=7.7$ $\mathrm{Hz}), 7.69$ (d, $1 \mathrm{H}, J=7.7 \mathrm{~Hz}), 7.86(\mathrm{~d}, 1 \mathrm{H}, J=7.7 \mathrm{~Hz}), 8.77$ (s, $1 \mathrm{H}) .{ }^{13} \mathrm{C} \mathrm{NMR}\left(100 \mathrm{MHz}, \mathrm{CDCl}_{3}\right): \delta=125.7,127.7$, 130.6, 131.3, 131.6, 132.5, 133.5, 136.2. MS (EI, $70 \mathrm{eV})$ : $m / z(\%)=309,307,305,262,260,258,228,226,198,196$, 182, 180 (100), 147, 101, 75, 50. Anal. Calcd for $\mathrm{C}_{8} \mathrm{H}_{5} \mathrm{Br}_{2} \mathrm{NO}_{2}$ (306.94): C, 31.30; $\mathrm{H}, 1.64 ; \mathrm{N}, 4.56$. Found: $\mathrm{C}$, $31.33 ; \mathrm{H}, 1.65 ; \mathrm{N}, 4.54$.

(Z)-5-(2-Bromo-2-nitrovinyl)-1,2,3-trimethoxybenzene (5j)

Orange solid, $\mathrm{mp} 113-115^{\circ} \mathrm{C}$. IR (neat): $v=3075,3035$, $1603,1577,1522,1333,1246,1125,824,618 \mathrm{~cm}^{-1} .{ }^{1} \mathrm{H}$ NMR (400 MHz, $\left.\mathrm{CDCl}_{3}\right): \delta=3.91(\mathrm{~s}, 6 \mathrm{H}), 3.93(\mathrm{~s}, 3 \mathrm{H})$, $7.19(\mathrm{~s}, 2 \mathrm{H}), 8.59(\mathrm{~s}, 1 \mathrm{H}) .{ }^{13} \mathrm{C}$ NMR $\left(100 \mathrm{MHz}, \mathrm{CDCl}_{3}\right): \delta$ $=56.4,56.5,61.3,105.9,108.9,125.2,127.1,131.9,136.7$, 141.8, 153.4. MS (EI, $70 \mathrm{eV}): m / z(\%)=319,317,272,270$, 257, 255, 192, 177 (100), 149, 134, 119, 63. Anal. Calcd for $\mathrm{C}_{11} \mathrm{H}_{12} \mathrm{BrNO}_{5}$ (318.12): C, 41.53; H, 3.80; N, 4.40. Found: $\mathrm{C}, 41.57 ; \mathrm{H}, 3.82 ; \mathrm{N}, 4.38$.

\section{(Z)-2-Bromo-5-(2-bromo-2-nitrovinyl)furan (5k)}

Yellow solid, $\mathrm{mp} 87-89^{\circ} \mathrm{C}$. IR (neat): $v=3134,3036,1618$, $1537,1505,1348,1292,1025,948,818,786,645 \mathrm{~cm}^{-1} .{ }^{1} \mathrm{H}$ $\operatorname{NMR}\left(400 \mathrm{MHz}, \mathrm{CDCl}_{3}\right): \delta=6.61(\mathrm{~d}, 1 \mathrm{H}, J=3.4 \mathrm{~Hz}), 7.37$ $(\mathrm{d}, 1 \mathrm{H}, J=3.4 \mathrm{~Hz}), 8.50(\mathrm{~s}, 1 \mathrm{H}) .{ }^{13} \mathrm{C}$ NMR $(100 \mathrm{MHz}$, $\left.\mathrm{CDCl}_{3}\right): \delta=115.8,122.3,124.0,125.1,129.6,148.6 . \mathrm{MS}$ $(\mathrm{EI}, 70 \mathrm{eV}): \mathrm{m} / z(\%)=299,297,295,225,223,221,135$, 133, 79, 63 (100). Anal. Calcd for $\mathrm{C}_{6} \mathrm{H}_{3} \mathrm{Br}_{2} \mathrm{NO}_{3}$ (296.90): C, 24.27; H, 1.02; N, 4.72. Found: C, 24.30; H, 1.03; N, 4.69. 